\title{
The impact of liver fat vs visceral fat in determining categories of prediabetes
}

\author{
K. Kantartzis • J. Machann • F. Schick • A. Fritsche • \\ H.-U. Häring • N. Stefan
}

Received: 18 September 2009 / Accepted: 17 December 2009/Published online: 23 January 2010

(C) Springer-Verlag 2010

\begin{abstract}
Aims/hypothesis Impaired fasting glucose (IFG) and impaired glucose tolerance (IGT) are risk factors for type 2 diabetes and cardiovascular disease; however, their impact on these endpoints differs. Because liver fat and visceral fat are important determinants of glucose and lipid metabolism, we investigated whether these fat compartments and their humoral products, the adipokine adiponectin and the hepatokine fetuin-A, differ in their impact on the glucose categories.

Methods In 330 individuals at risk of type 2 diabetes, glucose tolerance status was determined by a $2 \mathrm{~h} 75 \mathrm{~g}$ OGTT. Total-body and visceral fat were precisely quantified by magnetic resonance (MR) tomography and liver fat by ${ }^{1} \mathrm{H}-\mathrm{MR}$ spectroscopy.

Results A total of 210 individuals had normal glucose tolerance (NGT), 41 isolated IFG, 43 isolated IGT and 36 IFG+IGT. Total-body fat was not different $(p=0.51)$, although a small but continuous increase in visceral fat was found among the categories after adjustment for age and sex (NGT: $3.07 \pm 0.10 \mathrm{~kg}$; IFG: $3.11 \pm 0.21 \mathrm{~kg}$; IGT: $3.61 \pm 0.21 \mathrm{~kg}$; IFG + IGT: $3.84 \pm 0.23 \mathrm{~kg}$ [SEs], $p=0.03)$. A larger difference was found for liver fat (NGT: $4.73 \pm 0.42 \%$; IFG: $5.86 \pm 0.92 \%$; IGT: $8.65 \pm 0.92 \%$; IFG+IGT: $11.11 \pm 1.01 \%, p<0.0001$ ). The differences among the categories were small for
\end{abstract}

\footnotetext{
K. Kantartzis $\cdot$ A. Fritsche $\cdot$ H.-U. Häring $\cdot$ N. Stefan $(\triangle)$

Department of Internal Medicine, Division of Endocrinology, Diabetology, Vascular Medicine,

Nephrology and Clinical Chemistry, University of Tübingen, Otfried-Müller-Str. 10,

72076 Tübingen, Germany

e-mail: norbert.stefan@med.uni-tuebingen.de

J. Machann $\cdot$ F. Schick

Section of Experimental Radiology, University of Tübingen,

Tübingen, Germany
}

adiponectin $(p=0.14)$, but larger for fetuin-A $(p=0.015)$. Among fat compartments, liver fat $(p<0.0001)$ and among circulating variables fetuin-A $(p=0.016)$ were the strongest determinants of the categories.

Conclusions/interpretation Liver fat, more than visceral fat, strongly increases when glycaemia and glucose tolerance move from NGT to isolated IFG, isolated IGT and IFG+IGT. Because liver-derived circulating fetuin-A determines, although weakly, prediabetes categories, it is worth searching for hepatokines more strongly predicting prediabetes.

Keywords Adiponectin · Fetuin-A · Impaired fasting glucose $\cdot$ Impaired glucose tolerance $\cdot$ Liver fat .

Prediabetes $\cdot$ Visceral fat
Abbreviations
IFG Impaired fasting glucose
IGT Impaired glucose tolerance
MR Magnetic resonance
NGT Normal glucose tolerance

\section{Introduction}

The prediabetic states, impaired fasting glucose (IFG) and impaired glucose tolerance (IGT) most probably result from different pathophysiological mechanisms [1-4], although they are both associated with an increased risk of type 2 diabetes $[5,6]$. Furthermore, these prediabetic conditions are associated with an increased risk of cardiovascular disease and mortality. This was found in individuals with IFG+IGT and was shown for individuals with isolated IGT in most studies [7-9]. In contrast, individuals with isolated IFG have a modestly increased risk [10]. Of note, during 
the prediabetic state the increased risk appears to be dependent on the presence of cardiovascular risk factors such as hypercholesterolaemia and subclinical inflammation $[7,11]$. These variables are strongly related to visceral adiposity and fatty liver, established determinants of type 2 diabetes and cardiovascular disease [12-18].

Recently, we characterised metabolically benign obesity [19], a pathophysiologically very interesting condition [20]. In that study we identified low liver fat as the most important determinant of this phenotype, which is characterised by high insulin sensitivity and low intima-media thickness of the common carotid artery [19]. So far, the impact of liver fat vs visceral fat on determining the prediabetic conditions of isolated IFG, isolated IGT and IFG+IGT have not been investigated. Knowledge about the role of these fat compartments and their secreted products in the pathogenesis of prediabetes may help to develop both novel predictors of type 2 diabetes and individualised prevention and treatment strategies for impaired glucose and lipid metabolism.

Therefore, in the present study we investigated the relationships of total-body and visceral fat, precisely measured by magnetic resonance (MR) tomography, and ectopic fat deposition in liver and skeletal muscle, measured by ${ }^{1} \mathrm{H}-\mathrm{MR}$ spectroscopy, with glucose categories in a large cohort of 330 participants at risk of type 2 diabetes. To further corroborate the role of visceral fat and liver fat in determining the glucose categories, we investigated the relationships of the main humoral products of visceral fat and liver, adiponectin and fetuin-A [21, 22], which are determinants of incident type 2 diabetes and cardiovascular disease [23-27], with these categories.

\section{Methods}

Participants Data of 330 whites, 129 men and 201 women without type 2 diabetes, from the southern part of Germany, were analysed. These individuals were participating in an ongoing study to reduce adiposity and prevent type 2 diabetes [22, 28]. Individuals were included in the study when they fulfilled at least one of the following criteria: a family history of type 2 diabetes, a BMI $>27 \mathrm{~kg} / \mathrm{m}^{2}$ and a previous diagnosis of IGT and/or of gestational diabetes. They were considered healthy according to a physical examination and routine laboratory tests. The participants had no history of liver disease and did not consume more than two alcoholic drinks per day. Informed written consent was obtained from all participants and the local medical ethics committee approved the protocols.

Body fat distribution Waist circumference was measured at the midpoint between the lateral iliac crest and lowest rib.
Total-body and visceral fat were measured by MR tomography, with an axial T1-weighed fast spin echo technique with a $1.5 \mathrm{~T}$ whole-body imager (Magnetom Sonata; Siemens Medical Solutions, Erlangen, Germany), as previously described [29].

Liver fat and intramyocellular lipids Liver fat and intramyocellular fat of the tibialis anterior muscle were measured by localised ${ }^{1} \mathrm{H}-\mathrm{MR}$ spectroscopy, as previously described [29].

OGTT All individuals underwent a $75 \mathrm{~g}$ OGTT. We obtained venous plasma samples at $0,30,60,90$ and $120 \mathrm{~min}$ for determination of plasma glucose and insulin. Glucose tolerance was evaluated based on OGTT as follows: NGT (fasting glucose $<5.6 \mathrm{mmol} / \mathrm{l}$ and $2 \mathrm{~h}$ glucose $<7.8 \mathrm{mmol} / \mathrm{l}$ ); isolated IFG (fasting glucose $5.6-6.9 \mathrm{mmol} / \mathrm{l}$ and $2 \mathrm{~h}$ glucose $<7.8 \mathrm{mmol} / \mathrm{l}$ ); isolated IGT (fasting glucose $<5.6 \mathrm{mmol} / \mathrm{l}$ and $2 \mathrm{~h}$ glucose $7.8-11.0 \mathrm{mmol} / \mathrm{l}$ ); and IFG+IGT (fasting glucose $5.6-6.9 \mathrm{mmol} / 1$ and $2 \mathrm{~h}$ glucose 7.8 $11.0 \mathrm{mmol} / \mathrm{l})[30,31]$.

Insulin sensitivity from the OGTT was calculated as proposed by Matsuda and DeFronzo [32]. Furthermore, the HOMA insulin resistance index (HOMA-IR) was calculated [33]. Insulin secretion was assessed by calculating the incremental insulin/glucose ratio in the first $30 \mathrm{~min}$ of the OGTT $\left(\Delta \mathrm{Ins}_{30-0} / \Delta \mathrm{Glc}_{30-0}\right)$ and the AUC for insulin divided by the AUC for glucose during the $120 \mathrm{~min}$ of the test [34].

Euglycaemic-hyperinsulinaemic clamp A subgroup $(n=224)$ additionally underwent a euglycaemic-hyperinsulinaemic clamp. Insulin sensitivity was determined with a primed insulin infusion at a rate of $40 \mathrm{mU} \mathrm{m}{ }^{-2} \min ^{-1}$ for $2 \mathrm{~h}$, as previously described [29].

Analytical procedures Blood glucose was determined using a bedside glucose analyser (glucose oxidase method; Yellow Springs Instruments, Yellow Springs, CO, USA). Plasma insulin was determined by microparticle enzyme immunoassay (Abbott Laboratories, Tokyo, Japan). The plasma glucose and insulin AUCs during the OGTT were calculated using the trapezoidal rule. Plasma samples were frozen immediately and stored at $-80^{\circ} \mathrm{C}$. Fasting plasma levels of adiponectin were determined with ELISA (Linco Research, St Charles, MO, USA). Fasting plasma levels of fetuin-A were measured by an immunoturbidimetic method (BioVendor Laboratory Medicine, Modreci, Czech Republic), as previously described [24].

Statistical analyses Data are given as means \pm SE. Except waist circumference, which was normally distributed (Shapiro-Wilk $W$ test), all other data were not normally distributed and were $\log _{e}$-transformed. To test differences in 
body composition variables and metabolic characteristics among the four fasting and glucose tolerance categories, multivariate linear regression models were used with the variable to be considered set as the dependent variable. In these models the fasting and glucose tolerance categories were included as an independent nominal variable. To determine the predictive effect of total-body fat, visceral fat, liver fat, circulating adiponectin and circulating fetuin-A on the glucose tolerance categories, forward logistic stepwise regression analyses were performed. Furthermore, the OR for $1 \mathrm{SD}$ increase in visceral fat and liver fat of the individuals for having NGT or prediabetes (isolated IFG and/or isolated IGT and/or IFG+IGT) were calculated in nominal logistic regression analyses. Furthermore, using such a model the OR for having NGT or prediabetes was calculated for the presence or absence of fatty liver. $p \leq 0.05$ was considered statistically significant. The statistical software package JMP 5.1 (SAS Institute, Cary, NC, USA) was used.

\section{Results}

The 330 participants (129 men and 201 women) had a mean age of 45 (range 18-69) years. Their characteristics covered a wide range, e.g. BMI $19.36-46.98 \mathrm{~kg} / \mathrm{m}^{2}$; waist circumference $63-138 \mathrm{~cm}$; total-body fat $3.96-69.55 \mathrm{~kg}$; visceral fat $0.22-10.12 \mathrm{~kg}$ and liver fat $0.1-30.88 \%$. According to the cut-off of liver fat of 5.56\% [35], 104 individuals were found to have fatty liver.

Visceral fat was higher in men $(4.34 \pm 0.16$ vs $2.14 \pm$ $0.09 \mathrm{~kg}, p<0.0001)$ and correlated significantly with age $(r=0.36, p<0.0001)$, BMI $(r=0.62, p<0.0001)$, waist circumference $(r=0.82, p<0.0001)$, total-body fat $(r=0.45$, $p<0.0001)$ and liver fat $(r=0.60, p<0.0001)$ and weakly with intramyocellular fat $(r=0.11, p=0.06)$. Visceral fat adjusted for age, sex and total-body fat correlated inversely with insulin sensitivity (OGTT, $r=-0.29, p<0.0001$; clamp, $r=-0.32, p<0.0001)$ and circulating adiponectin $(r=-0.42$, $p<0.0001)$, but not with circulating fetuin-A $(r=0.08$, $p=0.19)$.

Liver fat was also higher in men $(7.31 \pm 0.60$ vs $4.89 \pm 0.42 \%$, $p<0.0001)$ and correlated significantly with age $(r=0.17$, $p=0.002)$, BMI $(r=0.44, p<0.0001)$, waist circumference $(r=0.53, p<0.0001)$, total-body fat $(r=0.31, p<0.0001)$ and intramyocellular fat $(r=0.20, p=0.0005)$. Liver fat adjusted for age, sex and total-body fat correlated inversely with insulin sensitivity (OGTT, $r=-0.44, p<0.0001$; clamp, $r=-0.47$, $p<0.0001)$ and circulating adiponectin $(r=-0.25, p<0.0001)$ and positively with circulating fetuin-A $(r=0.14, p=0.017)$.

Characteristics according to fasting glycaemia and glucose tolerance status Among the 330 participants, 210 were found to have NGT, 41 isolated IFG, 43 isolated IGT and 36 IFG+IGT (Table 1). Compared with the NGT group the percentage of men and age were higher in the IFG and IFG+IGT groups. Body weight, BMI, waist circumference and total-body fat were not different among the four categories after adjustment for age and sex. One of the inclusion criteria, in fact the most frequently applied, BMI $>27 \mathrm{~kg} / \mathrm{m}^{2}$, may partly account for the absence of a significant difference in total-body fat among the categories. However, there was a small but continuous increase in adjusted visceral fat from NGT to IFG+IGT (Fig. 1a) and a more pronounced increase in adjusted liver fat (Fig. 1b). In line, individuals with IGT or IFG+IGT more often had fatty liver than individuals with NGT or isolated IFG (Table 1).

Among the metabolic characteristics, insulin sensitivity adjusted for age, sex and total-body fat decreased from NGT to IFG+IGT. HOMA-IR was particularly high in individuals with IFG and IFG+IGT, while insulin sensitivity estimated from the OGTT and measured by the clamp showed a more continuous decrease from NGT to IFG + IGT. A similar trend was observed for insulin secretion adjusted for age, sex, total-body fat and insulin sensitivity estimated from the OGTT. Levels of the adipokine adiponectin were not different among the categories. Because adiponectin levels differ between men and women, analyses for adiponectin were also done separately in men and women. There was a small difference in adiponectin levels in women (NGT: $16.23 \pm 0.66 \mu \mathrm{g} / \mathrm{ml}$; IFG: $19.6 \pm 2.33 \mu \mathrm{g} / \mathrm{ml}$; IGT: $14.82 \pm 0.95 \mu \mathrm{g} / \mathrm{ml}$; IFG+IGT: $12.6 \pm 1.47 \mu \mathrm{g} / \mathrm{ml}, p=0.016$ ), whereas there was no difference in men $(p=0.09)$ among the categories. In all individuals there was a difference in the levels of the liver-derived protein fetuin-A (Fig. 1c, d). In this respect, the adjusted levels of fetuin-A were higher in individuals with isolated IGT compared with individuals with isolated IFG $(p<0.05)$.

Because of the number of tests performed a type I error may have occurred. However, because the variables tested in our study are highly correlated, and because we had a clear a priori hypothesis, namely that liver fat and its humoral product, fetuin-A, levels are more strongly correlated with prediabetic categories than visceral fat and its major humoral product, adiponectin, it is questionable whether a correction for multiple comparisons is appropriate. Nevertheless, when we corrected for the four tests performed, the relationships of liver fat and visceral fat with glucose categories remained significant also at the new $\alpha$ level of 0.0125 , while the relationship of circulating fetuin-A with the glucose categories was only of borderline significance $(p=0.015)$.

Predictive effect of the variables on fasting and glucose tolerance categories To investigate the impact of the fat compartments and their humoral signals in determining the 
Table 1 Participants' demographic and metabolic characteristics according to glucose tolerance status

\begin{tabular}{|c|c|c|c|c|c|}
\hline Characteristic & NGT & IFG & IGT & $\mathrm{IFG}+\mathrm{IGT}$ & $p$ value \\
\hline$n$ & 210 & 41 & 43 & 36 & \\
\hline \multicolumn{6}{|l|}{ Demographics } \\
\hline Sex, men $(\%)$ & 39 & 46 & 21 & 56 & $0.0098^{\mathrm{d}}$ \\
\hline Age (years) & $43.6 \pm 0.8$ & $47.1 \pm 1.7$ & $44.3 \pm 1.8$ & $54.8 \pm 1.6$ & $<0.0001$ \\
\hline \multicolumn{6}{|l|}{ Body composition } \\
\hline Body weight (kg) & $87.4 \pm 1.2$ & $85.9 \pm 2.7$ & $85.0 \pm 2.6$ & $88.5 \pm 1.9$ & 0.81 \\
\hline BMI $\left(\mathrm{kg} / \mathrm{m}^{2}\right)$ & $29.54 \pm 0.35$ & $28.99 \pm 0.71$ & $30.06 \pm 0.87$ & $30.89 \pm 0.72$ & 0.33 \\
\hline Waist circumference $(\mathrm{cm})$ & $96.02 \pm 0.92$ & $95.88 \pm 2.00$ & $96.51 \pm 2.14$ & $102.82 \pm 1.76$ & 0.17 \\
\hline Total-body fat (kg) & $26.54 \pm 0.76$ & $25.00 \pm 1.62$ & $28.12 \pm 1.69$ & $26.90 \pm 1.62$ & 0.51 \\
\hline Visceral fat (kg) & $2.76 \pm 0.12$ & $3.12 \pm 0.28$ & $2.95 \pm 0.28$ & $4.30 \pm 0.30$ & 0.03 \\
\hline Liver fat $(\%)$ & $4.43 \pm 0.32$ & $5.85 \pm 1.14$ & $7.97 \pm 1.14$ & $11.50 \pm 1.44$ & $<0.0001$ \\
\hline Fatty liver & $52(24.8 \%)$ & $9(22 \%)$ & $18(41.9 \%)$ & $25(69.4 \%)$ & $<0.0001^{\mathrm{d}}$ \\
\hline $\mathrm{IMCL}_{\text {Tibialis anterior }}\left(\right.$ arbitrary units) ${ }^{\mathrm{a}}$ & $3.84 \pm 0.13$ & $4.10 \pm 0.34$ & $4.55 \pm 0.30$ & $4.09 \pm 0.31$ & 0.34 \\
\hline \multicolumn{6}{|l|}{ Metabolic characteristics } \\
\hline Fasting glucose $(\mathrm{mmol} / \mathrm{l})$ & $5.01 \pm 0.02$ & $5.84 \pm 0.03$ & $5.04 \pm 0.05$ & $6.06 \pm 0.05$ & $<0.0001$ \\
\hline $2 \mathrm{~h}$ glucose $(\mathrm{mmol} / \mathrm{l})$ & $6.18 \pm 0.06$ & $6.25 \pm 0.13$ & $8.91 \pm 0.13$ & $9.42 \pm 0.19$ & $<0.0001$ \\
\hline Fasting insulin (pmol/1) & $57 \pm 2$ & $76 \pm 10$ & $63 \pm 6$ & $86 \pm 6$ & $<0.0001$ \\
\hline $2 \mathrm{~h}$ insulin $(\mathrm{pmol} / \mathrm{l})$ & $400 \pm 21$ & $441 \pm 56$ & $731 \pm 72$ & $907 \pm 77$ & $<0.0001$ \\
\hline HOMA-IR index & $1.74 \pm 0.08$ & $2.67 \pm 0.37$ & $1.92 \pm 0.18$ & $3.13 \pm 0.24$ & $<0.0001$ \\
\hline Insulin sensitivity ${ }_{\text {OGTT }}$ & $14.24 \pm 0.50$ & $11.32 \pm 0.96$ & $11.02 \pm 0.73$ & $6.97 \pm 0.56$ & $<0.0001$ \\
\hline $\mathrm{ISI}_{\text {clamp }}\left(\mu \mathrm{mol} \mathrm{kg}^{-1} \min ^{-1}\left[\mathrm{pmol} / 1^{-1}\right]\right)^{\mathrm{b}}$ & $0.070 \pm 0.003$ & $0.066 \pm 0.009$ & $0.052 \pm 0.003$ & $0.042 \pm 0.004$ & $<0.0001$ \\
\hline$\Delta \mathrm{Ins}_{30-0} / \Delta \mathrm{Glc}_{30-0}$ ratio $(\mathrm{pmol} / \mathrm{mmol})$ & $167 \pm 14$ & $129 \pm 13$ & $109 \pm 10$ & $99 \pm 11$ & $<0.0001$ \\
\hline $\mathrm{AUC}_{\mathrm{Ins} 120} / \mathrm{AUC}_{\mathrm{Glc} 120}(\mathrm{pmol} / \mathrm{mmol})$ & $65 \pm 3$ & $67 \pm 7$ & $60 \pm 6$ & $66 \pm 5$ & $<0.0001$ \\
\hline Fetuin-A $(\mu \mathrm{g} / \mathrm{ml})^{\mathrm{c}}$ & $269 \pm 4$ & $257 \pm 8$ & $288 \pm 9$ & $268 \pm 8$ & 0.015 \\
\hline Adiponectin $(\mu \mathrm{g} / \mathrm{ml})^{\mathrm{c}}$ & $14.0 \pm 0.5$ & $15.1 \pm 1.6$ & $13.4 \pm 0.9$ & $11.6 \pm 0.8$ & 0.14 \\
\hline
\end{tabular}

Values are unadjusted means \pm SE

For statistical analyses, non-normally distributed variables were log transformed. Body weight, BMI, waist circumference and total-body fat were adjusted for age and sex. The other variables were additionally adjusted for total-body fat. Insulin secretion indices were additionally adjusted for insulin sensitivity

IMCL, intramyocellular lipids, ISI, insulin sensitivity index

${ }^{\text {a }}$ Available in 291 individuals (NGT $n=184$; IFG $n=33$; IGT $n=43$; IFG+IGT $n=31$ )

${ }^{\mathrm{b}}$ Available in 224 individuals (NGT $n=147$; IFG $n=21$; IGT $n=31$; IFG+IGT $n=25$ )

${ }^{\mathrm{c}}$ Fetuin-A and adiponectin were available in 293 individuals (NGT $n=185$; IFG $n=37$, IGT $n=40$ IFG+IGT $n=31$ ).

${ }^{\mathrm{d}} \chi^{2}$ test

fasting glycaemia and glucose tolerance categories, we performed forward stepwise regression analysis. As independent variables we chose the variables age and sex as well as liver fat and visceral fat that were different among the categories and total-body fat, which is a determinant of visceral fat and liver fat. In a model including these variables, liver fat was the strongest determinant followed by age (Table 2 , model 1 ). In another model including age, sex, circulating adiponectin and circulating fetuin-A, age was the strongest determinant of the categories followed by fetuin-A and adiponectin (Table 2, model 2).

Next we investigated the predictive effects of the fat compartments and their humoral signals in determining fasting and $2 \mathrm{~h}$ glycaemia. In models including age, sex and total-body, visceral and liver fat, age $(p<0.0001)$, followed by liver fat $(p=0.01)$ and visceral fat $(p=0.03)$ were independent determinants of fasting glycaemia, while liver fat $(p<0.0001)$, followed by age $(p=0.0005)$ and sex $(p=0.01)$ were independent determinants of $2 \mathrm{~h}$ glycaemia. In models including age, sex, circulating adiponectin and circulating fetuin-A, only age $(p<0.0001)$ was an independent determinant of fasting glycaemia, while age $(p<0.0001)$ followed by fetuin-A $(p=0.0009)$, adiponectin $(p=0.0026)$ and sex $(p=0.02)$ were independent determinants of $2 \mathrm{~h}$ glycaemia.

Next we calculated the ORs of the participants for having prediabetes (IFG, IGT and IFG+IGT groups combined) vs NGT. Both before and after adjustment, among the fat compartments, again liver fat turned out to be 
Fig. 1 Visceral fat $(\mathbf{a}, p=0.03)$, liver fat $(\mathbf{b}, p<0.0001)$, circulating adiponectin (c, $p=0.14)$ and circulating fetuin-A $(\mathbf{d}, p=0.15)$ in individuals with NGT, IFG, IGT and IFG+IGT. Visceral fat and liver fat were adjusted for sex and age. Circulating adiponectin and fetuin-A were adjusted for sex, age and total-body fat a

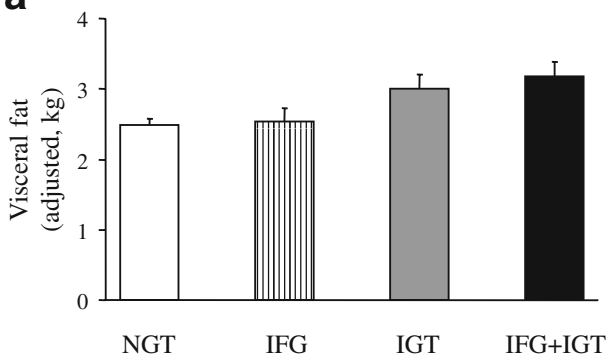

C

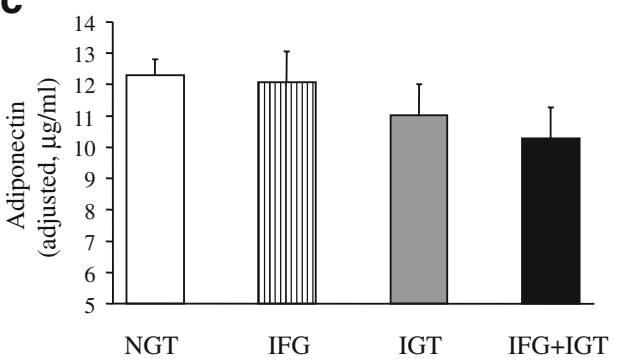

b

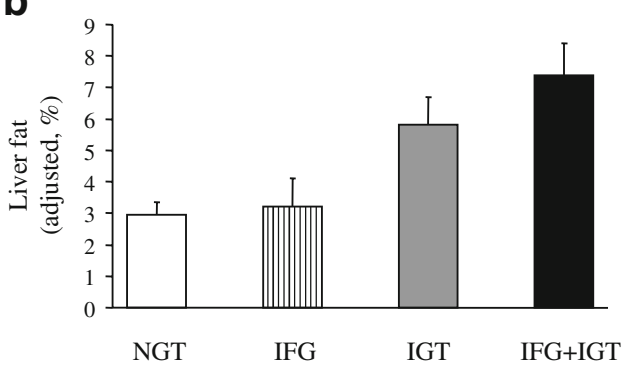

d

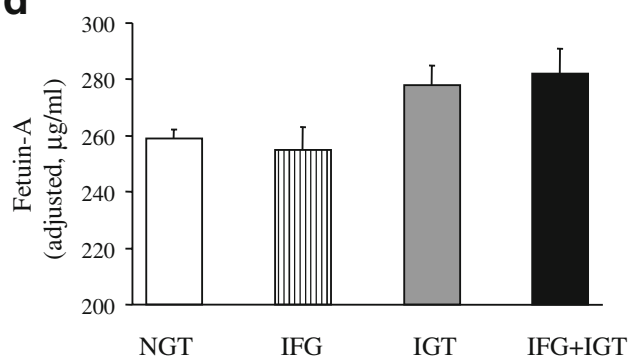

a stronger predictor than visceral fat. For a $1 \mathrm{SD}$ increase in liver fat the OR for having prediabetes was $1.82(95 \% \mathrm{CI}$ 1.44-2.33, Fig. 2a), and the adjusted OR was 1.78 (95\% CI $1.35-2.40$, Fig. 2b). The area under the receiver operating characteristic curve for liver fat predicting prediabetes was 0.65. Furthermore, individuals with fatty liver had an OR of 2.15 (95\% CI 1.39-3.23) and an adjusted OR of 1.80 (95\% CI 1.09-3.00) for having prediabetes. The positive predictive value of fatty liver for the diagnosis of prediabetes was 0.50 . The negative predictive value was 0.70 .

\section{Discussion}

In the present study, using precise measurements of body fat distribution, we found that the metabolically very active visceral fat continuously increases when fasting glycaemia

Table 2 Determinants of fasting glycaemia and glucose tolerance categories in a forward stepwise linear regression analysis

\begin{tabular}{lrc}
\hline Variable & Score $\chi^{2}$ & $p$ value \\
\hline Model 1 & & \\
Sex & 1.28 & 0.26 \\
Age & 11.12 & 0.0009 \\
Total-body fat & 0.74 & 0.39 \\
Visceral fat & 0.38 & 0.54 \\
Liver fat & 26.65 & $<0.0001$ \\
Model 2 & & \\
Sex & 1.99 & 0.16 \\
Age & 22.19 & $<0.0001$ \\
Adiponectin & 5.29 & 0.02 \\
Fetuin-A & 5.80 & 0.016 \\
\hline
\end{tabular}

and glucose tolerance move from NGT to isolated IFG, isolated IGT and IFG+IGT. This finding is in agreement with the recent data of Stancakova et al. [3], who found an increase in waist circumference, an estimate of visceral adiposity, in men among these prediabetic categories. However, we provide novel information that liver fat is a stronger determinant of prediabetic categories than visceral fat. These findings indicate that liver fat may be a more important determinant of impaired glucose regulation than visceral fat. Despite the fact that liver fat was found to be closely associated with visceral fat in many studies [17, 19, $21,28,36]$ and also in this report, the present data suggest that individuals may exist who have high liver fat but no increased visceral adiposity. When we applied the cut-off values for waist circumference indicating visceral obesity in men and women of the National Cholesterol Education Program's Adult Treatment Panel III report [37], we found indeed that $17 \%$ of our participants had fatty liver, but no visceral obesity. These data suggest that measuring liver fat may be useful in the determination of the diabetes risk even after measurement of waist circumference or visceral fat. In this aspect, a very recent study in 118 obese adolescents found a trend for an increase in the prevalence of prediabetes across tertiles of liver fat content. However, whether visceral or liver fat was more important in determining prediabetes was not studied [38].

To corroborate our findings, we investigated the relationships of the main humoral products of fat and liver, adiponectin and fetuin-A, with these categories. In agreement with the data about the fat compartments among the categories, the differences in circulating fetuin-A were larger than in circulating adiponectin, and fetuin-A was a somewhat stronger predictor of the categories than adiponectin. Thus, from a pathophysiological aspect, although both 


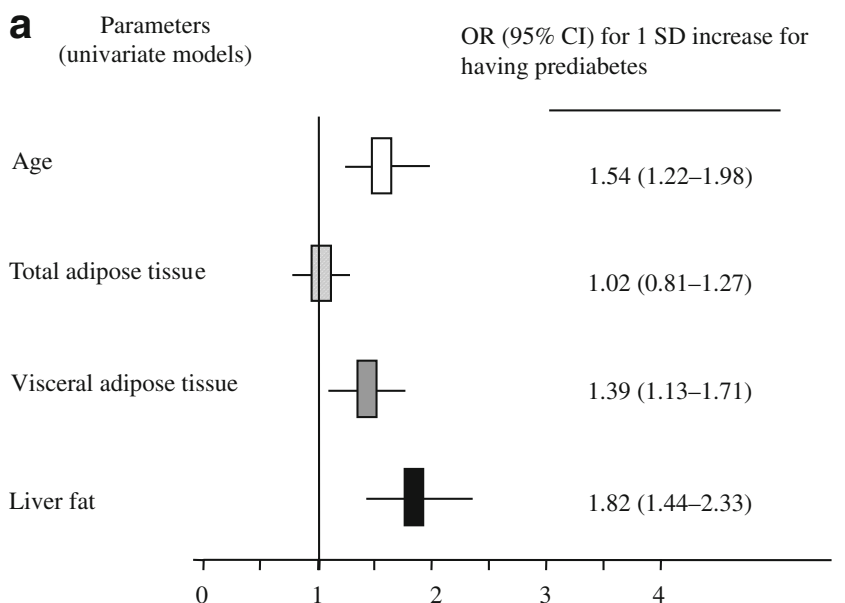

b $\begin{gathered}\text { Parameters } \\ \text { (multivariate model) }\end{gathered}$

OR $(95 \% \mathrm{CI})$ for $1 \mathrm{SD}$ increase for having prediabetes

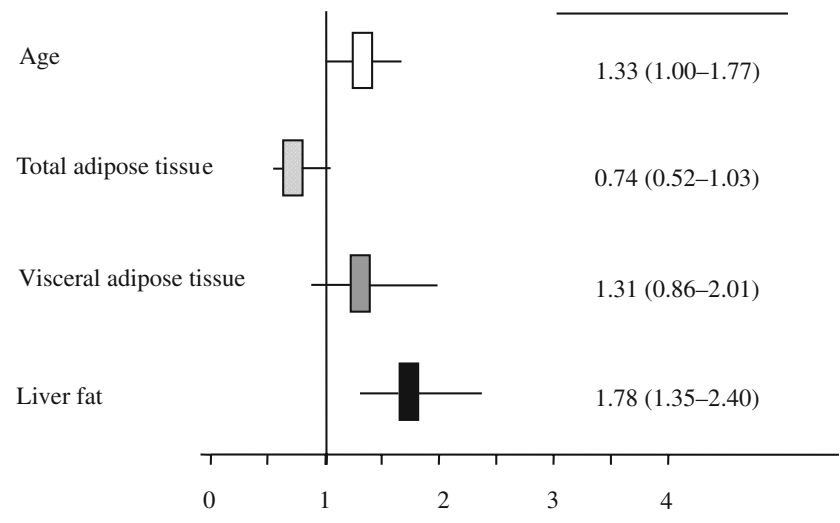

Fig. 2 ORs for 1 SD increase in age, total-body fat, visceral fat and liver fat, for having prediabetes compared with NGT in univariate analyses (a) and in multivariate analyses with additional adjustment for sex (b) (OR for women vs men: 0.37)

proteins are critical in the pathogenesis of diabetes and cardiovascular disease, it appears that targeting liver fat and its secreted products may be more promising than visceral fat in the prevention and treatment of impaired glucose metabolism.

How are liver and visceral fat related to abnormal glucose regulation? Both fat compartments are closely correlated with insulin resistance [14, 15, 39-41]. While effects of fatty liver in increasing hepatic glucose production via impaired hepatic insulin signalling are well established [42], its role as an endocrine organ has not been fully understood. The liver-secreted protein fetuin-A and the adipokine adiponectin appear to affect insulin sensitivity in different ways. Fetuin-A inhibits insulin receptor autophosphorylation, and thus has direct effects on insulin signalling [22]. Adiponectin mainly induces oxidation of ectopic fat in the liver and skeletal muscle, and via this mechanism protects from obesity/fat-induced insulin resistance [23]. In agreement with this hypothesis, human studies showed that circulating adiponectin correlated with insulin sensitivity particularly in more obese individuals [43]. If both circulating proteins have distinct and somewhat different effects on insulin sensitivity, then they should be independently correlated with insulin sensitivity. Indeed, in our study circulating fetuin-A and adiponectin were both strongly and independently correlated with insulin sensitivity estimated from the OGTT (both $p<0.0001$ ) and measured by the clamp (both $p<0.0001$ ). Furthermore, in more lean individuals the correlation of adiponectin with insulin sensitivity was weak (clamp, $p=0.02$ ) or absent (OGTT, $p=0.11$ ), while adiponectin strongly correlated with insulin sensitivity in more obese individuals (both $p<0.0001$ ). In contrast, similar strong correlations of circulating fetuin-A with insulin sensitivity were found among more obese and lean individuals.

Differences in insulin sensitivity among prediabetic categories have been reported in many studies. A continuous decrease in estimates of glucose disposal from NGT to IFG, IGT and IFG+IGT was found [1-3, 44]. In contrast, HOMA-IR derived from fasting insulin and glucose levels, and which largely represents insulin resistance of the liver, was found to be higher in individuals with isolated IFG than isolated IGT and highest in individuals with IFG+IGT $[2,3,44]$. In our study, the finding of higher liver fat, but lower HOMA-IR in individuals with isolated IGT compared with individuals with isolated IFG was somewhat unexpected. However, although liver fat closely correlates with hepatic insulin resistance, mechanisms other than hepatic fat may also be operative in the determination of hepatic insulin resistance in IFG. A lower insulin secretion for a given insulin sensitivity, as estimated by the disposition index, in individuals with isolated IGT may also explain the lower HOMA-IR in these individuals. Indeed, we found a significantly lower disposition index (incremental insulin response from $0-30 \mathrm{~min}$ of the OGTT $\times$ the insulin sensitivity measured by the clamp) in individuals with isolated IGT than IFG ( $4.72 \pm 0.92$ vs $6.55 \pm 1.12, p<0.05)$.

We next tested whether liver fat may also be associated with insulin secretion and, thereby, determine prediabetic categories. Differences in insulin secretion among the categories were reported in many studies $[2,3]$. In our study, liver fat did not correlate with estimates of insulin secretion adjusted for age, sex, adiposity and insulin sensitivity (all $p>0.28$ ), which is in agreement with other studies $[45,46]$. Thus, these data do not support the idea that liver fat determines the prediabetic categories via regulation of insulin secretion.

What do our present findings tell the clinician? We found a higher OR (1.82) for $1 \mathrm{SD}$ increase in liver fat than for 1 SD increase in visceral fat (1.39) for having prediabetes compared with having NGT. Furthermore, individuals with fatty liver had an OR of 2.15 for having 
prediabetes. Thus, there is a high chance that clinicians, suspecting fatty liver in their patients because of ultrasonographic results or laboratory tests, detect impaired glucose metabolism and/or diabetes when screening for these conditions. Of note, precise measurement of liver fat by ${ }^{1} \mathrm{H}-\mathrm{MR}$ spectroscopy is not feasible in clinical practice to date. Ultrasound is a much less sensitive method and it yields positive results only when liver fat exceeds $30 \%$ [47]. In the future, novel ultrasonographic methods are expected to provide more accurate results. Furthermore, although the circulating variables, adiponectin and fetuin-A, were found to be determinants of prediabetes, the predictive value was rather weak. Thus, more effort in searching for novel biomarkers of impaired glucose metabolism is needed. In addition, if liver fat is such an important determinant of prediabetic categories, then particularly reduction of liver fat would be expected to result in an improvement of impaired glucose metabolism. While treatment with thiazolidinediones is associated with a reduction in both liver and visceral fat content but with an increase in total-body fat [48], targeting peroxisome proliferator-activated receptor (PPAR)- $\delta$ appears to have specific effects on liver fat. Treatment with PPAR- $\delta$ agonists largely decreased liver fat, without an effect on adiposity [49]. In agreement, we found that single-nucleotide polymorphisms in the PPAR- $\delta$ gene are more strongly correlated with a reduction in liver fat than total-body or visceral fat during a lifestyle intervention [50]. Thus, drugs specifically increasing fat oxidation in the liver may have a large impact in the prevention and treatment of prediabetes.

The main advantage of the present study is the precise phenotyping using state-of-the-art methods in a relatively large cohort of non-diabetic individuals. However, in addition to the obvious limitation that our cohort included only whites, the cross-sectional nature of the study does not allow the drawing of definitive conclusions about causal relationships.

In conclusion, we found that liver fat predicted glucose tolerance categories more strongly than total and visceral fat. Thus, differences in liver fat may explain part of the different risk of IFG and IGT for the development of diabetes and cardiovascular disease. Furthermore, our data suggest that it is worth searching for hepatokines better than fetuin-A for predicting prediabetes. Finally, strategies aiming at reducing liver fat may represent an effective tool in the treatment of prediabetes and in the prevention of type 2 diabetes.

Acknowledgements The study was supported by a grant from the Deutsche Forschungsgemeinschaft (KFO 114) and the European Community's FP6 EUGENE6 (LSHM-CT-2004-512013). N. Stefan is currently supported by a Heisenberg-Grant of the Deutsche Forschungsgemeinschaft (STE-1096/1-1).
Duality of interest The authors declare that there is no duality of interest associated with this manuscript.

\section{References}

1. Laakso M, Zilinskaite J, Hansen T et al (2008) Insulin sensitivity, insulin release and glucagon-like peptide-1 levels in persons with impaired fasting glucose and/or impaired glucose tolerance in the EUGENE2 Study. Diabetologia 51:502-511

2. Abdul-Ghani MA, Tripathy D, DeFronzo RA (2006) Contributions of beta-cell dysfunction and insulin resistance to the pathogenesis of impaired glucose tolerance and impaired fasting glucose. Diabetes Care 29:1130-1139

3. Stancakova A, Javorsky M, Kuulasmaa T, Haffner SM, Kuusisto J, Laakso M (2009) Changes in insulin sensitivity and insulin release in relation to glycemia and glucose tolerance in 6,414 Finnish men. Diabetes 58:1212-1221

4. Meyer C, Pimenta W, Woerle HJ et al (2006) Different mechanisms for impaired fasting glucose and impaired postprandial glucose tolerance in humans. Diabetes Care 29:1909-1914

5. Nathan DM, Davidson MB, DeFronzo RA et al (2007) Impaired fasting glucose and impaired glucose tolerance: implications for care. Diabetes Care 30:753-759

6. Kim NH, Pavkov ME, Looker HC et al (2008) Plasma glucose regulation and mortality in pima Indians. Diabetes Care 31:488-492

7. Meigs JB, Nathan DM, D'Agostino RB Sr, Wilson PW (2002) Fasting and postchallenge glycemia and cardiovascular disease risk: the Framingham Offspring Study. Diabetes Care 25:18451850

8. Sorkin JD, Muller DC, Fleg JL, Andres R (2005) The relation of fasting and 2-h postchallenge plasma glucose concentrations to mortality: data from the Baltimore Longitudinal Study of Aging with a critical review of the literature. Diabetes Care 28:2626-2632

9. Balkau B, Hu G, Qiao Q, Tuomilehto J, Borch-Johnsen K, Pyorala K (2004) Prediction of the risk of cardiovascular mortality using a score that includes glucose as a risk factor. The DECODE Study. Diabetologia 47:2118-2128

10. Anon (1999) Glucose tolerance and mortality: comparison of WHO and American Diabetes Association diagnostic criteria. The DECODE Study Group. European Diabetes Epidemiology Group. Diabetes Epidemiology: Collaborative Analysis of Diagnostic Criteria in Europe. Lancet 354:617-621

11. Stern MP, Fatehi P, Williams K, Haffner SM (2002) Predicting future cardiovascular disease: do we need the oral glucose tolerance test? Diabetes Care 25:1851-1856

12. Wajchenberg BL (2000) Subcutaneous and visceral adipose tissue: their relation to the metabolic syndrome. Endocr Rev 21:697-738

13. Despres JP, Lemieux I (2006) Abdominal obesity and metabolic syndrome. Nature 444:881-887

14. Roden M (2006) Mechanisms of disease: hepatic steatosis in type 2 diabetes-pathogenesis and clinical relevance. Nat Clin Pract Endocrinol Metab 2:335-348

15. Stefan N, Kantartzis K, Haring HU (2008) Causes and metabolic consequences of fatty liver. Endocr Rev 29:939-960

16. Targher G, Bertolini L, Padovani R et al (2006) Relations between carotid artery wall thickness and liver histology in subjects with nonalcoholic fatty liver disease. Diabetes Care 29:1325-1330

17. Kotronen A, Yki-Jarvinen H (2008) Fatty liver: a novel component of the metabolic syndrome. Arterioscler Thromb Vasc Biol 28:27-38

18. Targher G, Marra F, Marchesini G (2008) Increased risk of cardiovascular disease in non-alcoholic fatty liver disease: causal effect or epiphenomenon? Diabetologia 51:1947-1953 
19. Stefan N, Kantartzis K, Machann J et al (2008) Identification and characterization of metabolically benign obesity in humans. Arch Intern Med 168:1609-1616

20. Karelis AD (2008) Metabolically healthy but obese individuals. Lancet 372:1281-1283

21. Kantartzis K, Rittig K, Balletshofer B et al (2006) The relationships of plasma adiponectin with a favorable lipid profile, decreased inflammation, and less ectopic fat accumulation depend on adiposity. Clin Chem 52:1934-1942

22. Stefan N, Hennige AM, Staiger H et al (2006) Alpha2-HeremansSchmid glycoprotein/fetuin-A is associated with insulin resistance and fat accumulation in the liver in humans. Diabetes Care 29:853-857

23. Trujillo ME, Scherer PE (2006) Adipose tissue-derived factors: impact on health and disease. Endocr Rev 27:762-778

24. Stefan N, Fritsche A, Weikert C et al (2008) Plasma fetuin-A levels and the risk of type 2 diabetes. Diabetes 57:2762-2767

25. Weikert C, Stefan N, Schulze MB et al (2008) Plasma fetuin-a levels and the risk of myocardial infarction and ischemic stroke. Circulation 118:2555-2562

26. Ix JH, Wassel CL, Kanaya AM et al (2008) Fetuin-A and incident diabetes mellitus in older persons. JAMA 300:182-188

27. Fisher E, Stefan N, Saar K et al (2009) Association of AHSG gene polymorphisms with fetuin-A plasma levels and cardiovascular diseases in the EPIC-Potsdam Study. Circ Cardiovasc Genet 2:607-613

28. Kantartzis K, Thamer C, Peter A et al (2009) High cardiorespiratory fitness is an independent predictor of the reduction in liver fat during a lifestyle intervention in non-alcoholic fatty liver disease. Gut 58:1281-1288

29. Stefan N, Machicao F, Staiger H et al (2005) Polymorphisms in the gene encoding adiponectin receptor 1 are associated with insulin resistance and high liver fat. Diabetologia 48:2282-2291

30. Genuth S, Alberti KG, Bennett P et al (2003) Follow-up report on the diagnosis of diabetes mellitus. Diabetes Care 26:3160-3167

31. Forouhi NG, Balkau B, Borch-Johnsen K et al (2006) The threshold for diagnosing impaired fasting glucose: a position statement by the European Diabetes Epidemiology Group. Diabetologia 49:822-827

32. Matsuda M, DeFronzo RA (1999) Insulin sensitivity indices obtained from oral glucose tolerance testing: comparison with the euglycemic insulin clamp. Diabetes Care 22:1462-1470

33. Matthews DR, Hosker JP, Rudenski AS, Naylor BA, Treacher DF, Turner RC (1985) Homeostasis model assessment: insulin resistance and beta-cell function from fasting plasma glucose and insulin concentrations in man. Diabetologia 28:412-419

34. Staiger H, Machicao F, Kantartzis K et al (2008) Novel metaanalysis-derived type 2 diabetes risk loci do not determine prediabetic phenotypes. PLoS ONE 3:e3019

35. Szczepaniak LS, Nurenberg P, Leonard D et al (2005) Magnetic resonance spectroscopy to measure hepatic triglyceride content: prevalence of hepatic steatosis in the general population. Am J Physiol Endocrinol Metab 288:E462-E468
36. Kelley DE, McKolanis TM, Hegazi RA, Kuller LH, Kalhan SC (2003) Fatty liver in type 2 diabetes mellitus: relation to regional adiposity, fatty acids, and insulin resistance. Am J Physiol Endocrinol Metab 285:E906-E916

37. Grundy SM, Cleeman JI, Daniels SR et al (2005) Diagnosis and management of the metabolic syndrome: an American Heart Association/National Heart, Lung, and Blood Institute scientific statement. Circulation 112:2735-2752

38. Cali AM, de Oliveira AM, Kim H et al (2009) Glucose dysregulation and hepatic steatosis in obese adolescents: is there a link? Hepatology 49:1896-1903

39. Utzschneider KM, Kahn SE (2006) Review: the role of insulin resistance in nonalcoholic fatty liver disease. J Clin Endocrinol Metab 91:4753-4761

40. Szendroedi J, Roden M (2009) Ectopic lipids and organ function. Curr Opin Lipidol 20:50-56

41. Perseghin G, Bonfanti R, Magni S et al (2006) Insulin resistance and whole body energy homeostasis in obese adolescents with fatty liver disease. Am J Physiol Endocrinol Metab 291:E697E703

42. Samuel VT, Liu ZX, Qu X et al (2004) Mechanism of hepatic insulin resistance in non-alcoholic fatty liver disease. J Biol Chem 279:32345-32353

43. Kantartzis K, Fritsche A, Tschritter O et al (2005) The association between plasma adiponectin and insulin sensitivity in humans depends on obesity. Obes Res 13:1683-1691

44. Faerch K, Vaag A, Holst JJ, Hansen T, Jorgensen T, BorchJohnsen K (2009) Natural history of insulin sensitivity and insulin secretion in the progression from normal glucose tolerance to impaired fasting glycemia and impaired glucose tolerance: the Inter99 Study. Diabetes Care 32:439-444

45. Tushuizen ME, Bunck MC, Pouwels PJ, Bontemps S, Mari A, Diamant M (2008) Lack of association of liver fat with model parameters of beta-cell function in men with impaired glucose tolerance and type 2 diabetes. Eur J Endocrinol 159:251-257

46. Rijkelijkhuizen JM, Doesburg T, Girman CJ et al (2009) Hepatic fat is not associated with beta-cell function or postprandial free fatty acid response. Metabolism 58:196-203

47. McCullough AJ (2004) The clinical features, diagnosis and natural history of nonalcoholic fatty liver disease. Clin Liver Dis $8: 521-533$, viii

48. Belfort R, Harrison SA, Brown K et al (2006) A placebocontrolled trial of pioglitazone in subjects with nonalcoholic steatohepatitis. N Engl J Med 355:2297-2307

49. Riserus U, Sprecher D, Johnson T et al (2008) Activation of peroxisome proliferator-activated receptor (PPAR)delta promotes reversal of multiple metabolic abnormalities, reduces oxidative stress, and increases fatty acid oxidation in moderately obese men. Diabetes 57:332-339

50. Thamer C, Machann J, Stefan N et al (2008) Variations in PPARD determine the change in body composition during lifestyle intervention: a whole-body magnetic resonance study. J Clin Endocrinol Metab 93:1497-1500 